\title{
Safety and feasibility of multiple blood-brain barrier disruptions for the treatment of glioblastoma in patients undergoing standard adjuvant chemotherapy
}

\author{
So Hee Park, MD, ${ }^{1}$ Myung Ji Kim, MD, ${ }^{1}$ Hyun Ho Jung, MD, PhD, ${ }^{1}$ Won Seok Chang, MD, PhD, ${ }^{1}$ \\ Hyun Seok Choi, MD, PhD, ${ }^{2}$ Itay Rachmilevitch, BSc, ${ }^{3}$ Eyal Zadicario, PhD, ${ }^{3}$ and \\ Jin Woo Chang, MD, PhD'
}

${ }^{1}$ Brain Research Institute, Department of Neurosurgery and 'Department of Radiology, Yonsei University College of Medicine, Seoul, Korea; and ${ }^{3}$ InSightec, Ltd., Haifa, Israel

OBJECTIVE Glioblastoma (GBM) remains fatal due to the blood-brain barrier (BBB), which interferes with the delivery of chemotherapeutic agents. The purpose of this study was to evaluate the safety and feasibility of repeated disruption of the BBB (BBBD) with MR-guided focused ultrasound (MRgFUS) in patients with GBM during standard adjuvant temozolomide (TMZ) chemotherapy.

METHODS This study was a prospective, single-center, single-arm study. BBBD with MRgFUS was performed adjacent to the tumor resection margin on the 1st or 2nd day of the adjuvant TMZ chemotherapy at the same targets for 6 cycles. T2*-weighted/gradient echo (GRE) MRI was performed immediately after every sonication trial, and comprehensive MRI was performed at the completion of all sonication sessions. Radiological, laboratory, and clinical evaluations were performed 2 days before each planned BBBD.

RESULTS From September 2018, 6 patients underwent 145 BBBD trials at various locations in the brain. The authors observed gadolinium-enhancing spots at the site of BBBD on T1-weighted MRI in 131 trials (90.3\%) and 93 trials (64.1\%) showed similar spots on T2*-weighted/GRE MRI. When the 2 sequences were combined, BBBD was observed in 134 targets $(92.4 \%)$. The spots disappeared on follow-up MRI. There were no imaging changes related to BBBD and no clinical adverse effects during the 6 cycles.

CONCLUSIONS This study is the first in which repetitive MRgFUS was performed at the same targets with a standard chemotherapy protocol for malignant brain tumor. BBBD with MRgFUS was performed accurately, repeatedly, and safely. Although a longer follow-up period is needed, this study allows for the possibility of other therapeutic agents that previously could not be used due to the BBB.

Clinical trial registration no.: NCT03712293 (clinicaltrials.gov)

https://thejns.org/doi/abs/10.3171/2019.10.JNS192206

KEYWORDS magnetic resonance imaging; focused ultrasound; glioblastoma; blood-brain barrier; surgical technique; oncology

$\mathrm{G}$ Lioblastoma (GBM) is a diffuse and infiltrative glial tumor. It is the most common and aggressive primary brain tumor, accounting for more than half of all gliomas and $15 \%$ of all primary central nervous system tumors. ${ }^{15}$ The standard treatment regimen consists of surgery and concomitant chemoradiotherapy with 6 cycles of adjuvant temozolomide (TMZ) chemotherapy. ${ }^{22}$
This treatment has increased the median survival of GBM patients to 14.6 months, with a 2-year survival of $27.2 \%$ and a 5-year survival of $10 \% .^{21,22}$

Major causes of death due to GBM after treatment are tumor recurrence and progression. Tumor recurrence usually develops within $2 \mathrm{~cm}$ of the original lesion. ${ }^{6}$ This area is commonly infiltrated with tumor cells, but the blood-

ABBREVIATIONS AU = arbitrary unit; $\mathrm{BBB}=$ blood-brain barrier; $\mathrm{BBBD}=$ disruption of the $\mathrm{BBB} ; \mathrm{GBM}=$ glioblastoma; $\mathrm{GRE}=$ gradient echo; $\mathrm{MB}=$ microbubble; MRgFUS = MR-guided focused ultrasound; P-gp = P-glycoprotein 1; TMZ = temozolomide.

SUBMITTED August 15, 2019. ACCEPTED October 14, 2019.

INCLUDE WHEN CITING Published online January 3, 2020; DOI: 10.3171/2019.10.JNS192206. 
TABLE 1. Inclusion and exclusion criteria

\begin{tabular}{l}
\hline Inclusion criteria \\
\hline Male or female btwn 18 \& 80 yrs old \\
Grade IV malignant glioma confirmed through assessment of surgi- \\
cal specimens by neuropathologist \\
Underwent maximal safe resection \& completed TMZ chemothera- \\
py \& concurrent radiotherapy w/o any complication \& eligible for \\
adjuvant TMZ treatment \\
Tumor resection cavity is clearly defined on T1WI \& FLAIR se- \\
quence of MRI \\
Karnofsky Performance Scale score $70-100$ \\
\hline Exclusion criteria \\
Contraindications to MRI, MRI contrast, or ultrasound contrast \\
MRI findings of brain edema \&/or mass effect requiring steroid \\
treatment, hemorrhage, calcified lesion \\
$\geq 30 \%$ of the skull area traversed by sonication is covered by scars, \\
scalp disorder, or atrophic scalp \\
Receiving other concurrent therapies such as bevacizumab therapy, \\
immunotherapies, stereotactic radiosurgery, wafer, convection- \\
enhanced delivery \\
Significant cardiac disease, uncontrolled hypertension \\
Predisposition for bleeding \\
Known cerebral or systemic vasculopathy \\
Uncontrolled insulin-dependent diabetes mellitus \\
Impaired liver/renal function \\
Severe chronic respiratory disorders \\
Evidence of cranial or systemic infection
\end{tabular}

T1WI = T1-weighted imaging.

brain barrier (BBB) in this region is mostly intact. Various strategies to overcome the BBB have been investigated. However, these approaches have been limited by the lack of specificity, safety concerns, and a failure to deliver adequate concentrations of therapeutic agents to brain tissue. ${ }^{20}$

Recently, MR-guided focused ultrasound (MRgFUS) has been attracting attention as a noninvasive means of temporarily disrupting the BBB. MRgFUS delivers ultrasound energy to the target with intraoperative imaging guidance and real-time feedback for monitoring without opening the scalp or skull. Comprising a phased-array transducer system of 1024 individually steered elements, the current MRgFUS device can target brain regions with submillimetric accuracy. ${ }^{7}$ Low-intensity focused ultrasound (FUS) disrupts the BBB using the cavitation effect, and the addition of microbubbles (MBs) lowers the cavitation threshold, thereby enabling a reduction in the acoustic power needed for disruption of the BBB (BBBD). This reduction in acoustic energy allows safe transcranial ultrasound administration..$^{18}$

Currently, studies are underway to determine if BBBD can be safely achieved in patients. ${ }^{9,11}$ Therefore, this study aimed to investigate whether BBBD with MRgFUS could be repeatedly and safely performed in patients who underwent surgery for GBM.

\section{Methods \\ Clinical Trial Design}

This study was a prospective, single-center, singlearm study (clinical trial registration no.: NCT03712293 [clinicaltrials.gov]). Patients were enrolled after resection of their GBM and radiotherapy with concomitant TMZ chemotherapy and prior to their planned adjuvant TMZ chemotherapy. Included patients underwent gross-total resection of their tumor, which was confirmed as a WHO grade IV malignant glioma by a neuropathologist. Detailed inclusion and exclusion criteria are listed in Table 1.

TMZ chemotherapy was performed according to the standard protocol. BBBD was performed on the 1st or 2nd day of the 4-week chemotherapy cycle for 6 cycles. Targets for sonication were within $2 \mathrm{~cm}$ of the tumor margin and selected mainly on the white matter that had a high signal on the FLAIR image. To avoid overlapping the sonicated area, the distance between targets was more than 1 $\mathrm{cm}$. Targets were planned with presonication MRI and selected by the neurosurgeons and neuroradiologists. BBBD was performed on the same targets at every cycle.

Radiological evaluation was performed 2 days before and immediately after each planned BBBD cycle. Laboratory testing was performed 2 days before each planned BBBD cycle, and clinical neurological evaluation was performed 2 days before and 1 day after each planned BBBD cycle. Tumor status was evaluated based on MRI findings according to the RANO (Response Assessment in NeuroOncology) criteria. If tumor progression was observed, the patient exited the trial and received alternative therapies. Figure 1 outlines the study design. This study was approved by our institutional review board, and patient informed consent was obtained.

\section{Procedure for BBBD}

\section{Patient Preparation and MRI Planning}

On the day of the procedure, an 18-gauge intravenous catheter was inserted into the patient's left forearm vein.

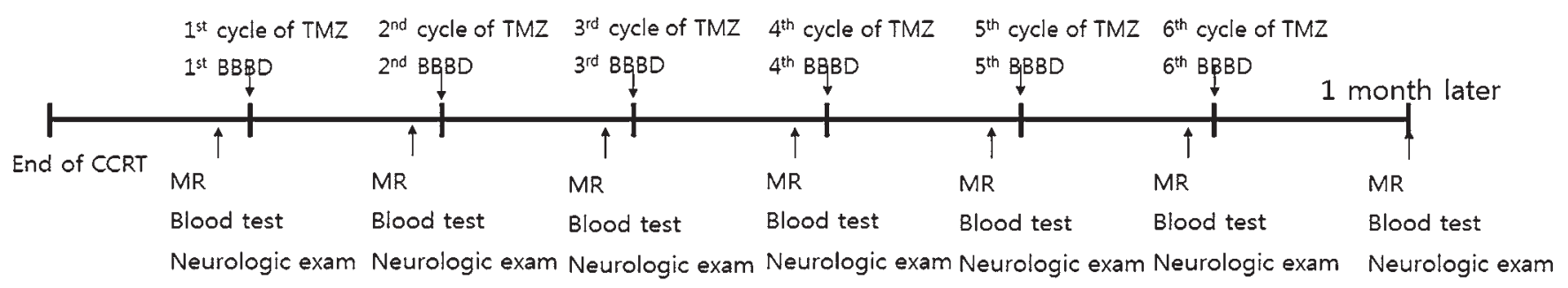

FIG. 1. Overview of the study design. CCRT = concurrent chemoradiation therapy. 

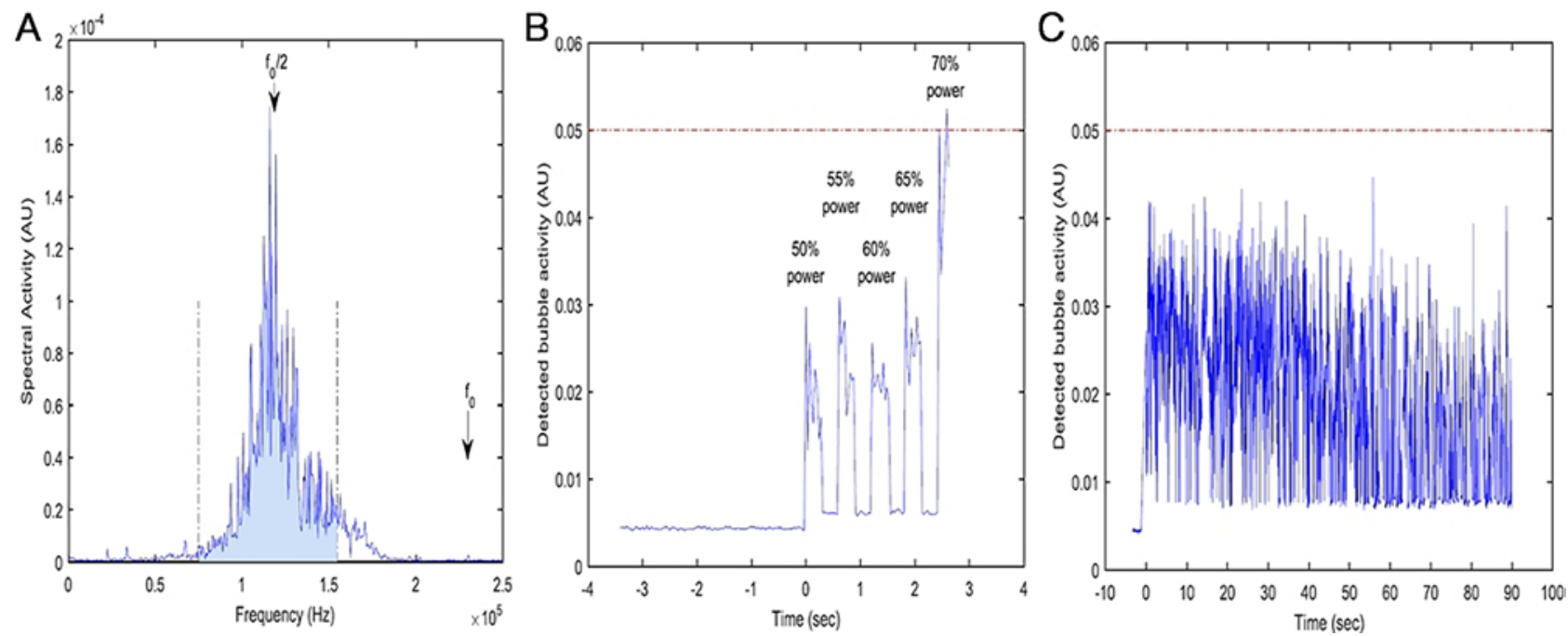

FIG. 2. Graph of spectral activity and detected bubble activity. A: Typical spectral activity detected in the system's hydrophones. Spectral density was plotted against frequency (solid blue lines). Hydrophone data detected at each ultrasound pulse were used to calculate the broadband subharmonic signal. Bubble activity was calculated with the integrated spectral density from 75 to 155 $\mathrm{kHz}$ (area under blue curve between the two dotted black lines). B: Ramp sonication of cycle 3 in patient 4 . The requested power was $11 \mathrm{~W}$, and ramp sonication started at $50 \%$ of the requested power $(5.5 \mathrm{~W})$. Bubble activity was plotted against time while the power was gradually increased from $50 \%$ to full power in $5 \%$ intervals approximately every 0.3 second in a stepwise manner (blue lines). Bubble activity was monitored, and the power ramp was stopped when the activity exceeded a predefined value (red dotted line). C: Detected bubble activity during sonication in cycle 4 in patient 1 . Sonication was performed at $50 \%$ of the power level where ramp sonication was stopped. Bubble activity was plotted versus time during a 90 -second treatment sonication (blue lines). The power was adjusted to achieve sufficient activity yet not to exceed a predefined "danger" level (red dotted line). Figure is available in color online only.

The patient's head was shaved and chemically depilated. A Cosman-Roberts-Wells stereotactic frame was fixed to the patient's head under local anesthesia.

For imaging guidance, a 3.0-T MRI unit (Signa MR750, GE Healthcare) was used. First, a T1-weighted 3D scan was obtained. Subsequently, to optimize the quality of MRI due to the distance from the MR coil to the patient's head, a B1 map was acquired to adjust the transmit gain. A T2-weighted planning image was obtained, and the images were coregistered with presonication MRI for precise targeting.

\section{Sonication}

BBBD was performed using MRgFUS (ExAblate Neuro Model 4000 Type 2.0 [220 kHz] system, InSightec). Ultrasound pulse sequences were delivered to an array of 3 $\times 3$ subspots, arranged on a 3-mm-spaced subspot grid in a cyclic sequential manner, repeating the cycle every 2.7 seconds. For each subspot, a pulse train of $10 \times 2.6-\mathrm{msec}-$ long pulses with a 30.4-msec interval between pulses was used.

Such sonication sessions were coordinated with intravenous injection of MBs (Definity [perflutren lipid microsphere], Lantheus Medical Imaging, Inc.). The maximum dose of MB agent was $20 \mu \mathrm{l} / \mathrm{kg}$. The dose of single injections was $4 \mu \mathrm{l} / \mathrm{kg}$; therefore, a total of 5 injections were possible. A $4-\mu \mathrm{l} / \mathrm{kg}$ bolus of Definity was injected for 10 seconds, and then saline irrigation was performed for 10 seconds. Before each sonication trial, a dummy sonication ( $2 \mathrm{~W}$ for 2 seconds) was performed to estimate system time delays and achieve correct MB injection-sonication timing (typically, a 10- to 15-second delay postinjection was needed).

During sonication, bubble activity was monitored and recorded by hydrophones embedded in the system. The subharmonic broadband signal, integrating the spectral density from $75 \mathrm{kHz}$ to $155 \mathrm{kHz}, 80 \mathrm{kHz}$ around half of the ultrasound frequency, was seen as bubble activity (Fig. 2A). For safety, optimal power for BBBD was calculated as $50 \%$ of the power level at which bubble activities were high enough to generate significant inertial cavitation. This power was deduced from the "ramp sonication," which was designed to gradually increase the power from $50 \%$ to full power in 5\% intervals every 0.3 second until the detected bubble activity reached a predefined level that was enough to generate inertial cavitation and halt (Fig. 2B). Once the required power was established, 90 -secondlong sonication was used for BBBD. Bubble activity of the subharmonic broadband signal was monitored during sonication, and the accumulated bubble activity (that is, the spectral dose) was used to assess the treatment (Fig. 2C). Repeated sonication with incrementally adjusted power levels was used to reach a sufficient level of accumulated bubble activity and disrupt the BBB. The maximum power level used did not exceed $40 \mathrm{~W}$. Sonication was applied to the target during a treatment time window of 10 minutes after each bolus of Definity. If necessary, another bolus of Definity was administered.

\section{Evaluation of Safety and Efficacy of BBBD With MRgFUS}

The safety issues of repeated multiple disruptions of the BBB were investigated by radiological, laboratory, and 
TABLE 2. Patient demographics

\begin{tabular}{ccllccccc}
\hline Patient No. & Age (yrs), Sex & Location & IDH & MGMT & 1p19q Codeletion & EGFR & Neurological Status & Skull Density Ratio \\
\hline 1 & $53, \mathrm{~F}$ & Lt frontal & Wild & + & - & - & No deficit & 0.31 \\
\hline 2 & $62, \mathrm{M}$ & Rt temporal & Wild & + & - & - & No deficit & 0.41 \\
\hline 3 & $53, \mathrm{M}$ & Rt parietal & Wild & - & - & - & No deficit & 0.36 \\
\hline 4 & $67, \mathrm{M}$ & Lt temporal & Wild & + & - & - & No deficit & 0.37 \\
\hline 5 & $50, \mathrm{M}$ & Lt parietal & Wild & + & - & - & No deficit & 0.45 \\
\hline 6 & $50, \mathrm{~F}$ & Rt parietal & Wild & - & - & - & No deficit & 0.46 \\
\hline
\end{tabular}

$\mathrm{IDH}=$ isocitrate dehydrogenase; $\mathrm{MGMT}=$ methylguanine-DNA methyltransferase; EGFR = epidermal growth factor receptor; + = positive; $-=$ negative.

clinical evaluations. T2*-weighted/gradient echo (GRE) MRI sequences were obtained immediately after each sonication trial, and postsonication MRI was performed at the end of each session to evaluate any possible sign of hemorrhage, edema, or any other adverse radiological effects. Presonication MRI was performed 2-3 days before treatment to evaluate the delayed radiological adverse events and assess tumor status. Through regular laboratory and clinical evaluations at each cycle, adverse events that could be caused by BBBD were monitored.

Feasibility and efficacy of BBBD were evaluated through assessment of postsonication contrast-enhanced MRI in comparison with presonication MRI. The absolute concentration of gadolinium leakage cannot be measured by MRI techniques. Therefore, relative increases in signal intensity within the sonicated region were rated by an independent neurosurgeon and a neuroradiologist. Among the 9 grid points of the array of $3 \times 3$ subspots, when the number of visually recognizable spots was more than 5 (of 9), the sonicated region was determined as BBBD.

\section{Results}

From September 2018, 6 patients (4 men and 2 women, mean age $55.83 \pm 11.03$ years) were enrolled in the study. Patient demographics are shown in Table 2. Patient 4 dropped out of the study after the third cycle of BBBD. He had a GBM on the left parietal area and exhibited a minor personality issue before enrollment; he also refused to continue with the trials in the middle of the study.

Tumor locations were varied (frontal in 1 patient, temporal in 2, and parietal in 3). A total of 33 sessions were performed in 6 patients. Due to the limitation of the number of MBs, the maximum number of sessions was 6 (mean $4.4 \pm 0.9$ ) per cycle, and a total of 145 trials were performed in 6 patients. BBBD was performed 82 times in the right hemisphere and 63 times in the left hemisphere. BBBD was performed in a region adjacent to eloquent area in 5 patients at 1 or 2 sites (a total of 28 trials) during each BBBD procedure.

BBBD was seen on T1-weighted or T2*-weighted/GRE MRI. On T1-weighted MRI, the BBBD targets exhibited a bright signal intensity with 9 visible spots at the sonication area of $1 \mathrm{~cm}^{3}$ (Fig. 3). Among 145 targets, we observed gadolinium enhancement in 131 (90.3\%). Among all BBBD targets, 93 targets (64.1\%) exhibited dark signal spots on T2*-weighted/GRE MRI. However, the change in signal intensity did not always correspond to the enhance- ment of target areas with gadolinium (Table 3). It was possible for spots to be seen on T1-weighted but not on T2*-weighted/GRE sequences, on T2*-weighted/GRE but not on T1-weighted sequences, or on both. When we combined T1-weighted and T2*-weighted/GRE MRI, BBBD was observed at 134 targets (92.4\%) (Table 3). Usually, these spots disappeared on the follow-up MRI before the next BBBD session (Fig. 3).

The mean time from MB injection start to ramp sonication start was $13.86 \pm 9.19$ seconds $( \pm \mathrm{SD})$, and the mean time from MB injection start to sonication start was 96.65 \pm 55.60 seconds. The average number of sonications was 2.65 per target, and the average number of sonications over 30 seconds was 2.43 per target. Each sonication was performed for an average of 79.89 seconds. Except for 31 sonications that were stopped earlier than 30 seconds, sonication was performed for an average of 86.27 seconds. The most common reason for stopping sonication earlier than 30 seconds was due to its high power setting (23/31 cases). Most of these cases were distributed in the early trials of patient 1 . The other reasons included a low setting of sonication power in 5 cases, high spectral dose in 2 cases, and stopping for a mechanical check in 1 case. The mean time between sonications was $131.14 \pm 41.68$ seconds. Sonication was stopped within 30 seconds in 31 sonications, and there was target movement in 8 cases. Excluding these 39 cases, the time between sonication was $139.37 \pm 24.72$ seconds.

The mean starting sonication power of the first sonication performed over 30 seconds was $6.28 \pm 2.19 \mathrm{~W}$. On average, the starting sonication power was $50.77 \%$ of the ramp sonication power. The mean power of ramp sonication, the first sonication performed over 30 seconds, and the spectral dose for 6 cycles in 6 patients are shown in Table 4. Except for the early trials, there was no significant difference in the ramp sonication power and start sonication power between cycles in the same patient. The spectral dose of the target where BBBD was observed on both T1-weighted and T2*-weighted/GRE images was $10.80 \pm$ 4.36 arbitrary units (AU), that of the target where BBBD was observed in either MR sequence was $10.21 \pm 4.15$ $\mathrm{AU}$, and that of the target where BBBD was observed on neither T1-weighted nor T2*-weighted/GRE images was $7.81 \pm 4.16$ AU. When BBBD was observed on both MR sequences, the spectral dose was about 1.4 times higher than when it was not seen on both MR sequences, without reaching statistical significance. These results showed that a high spectral dose is associated with BBBD. The 

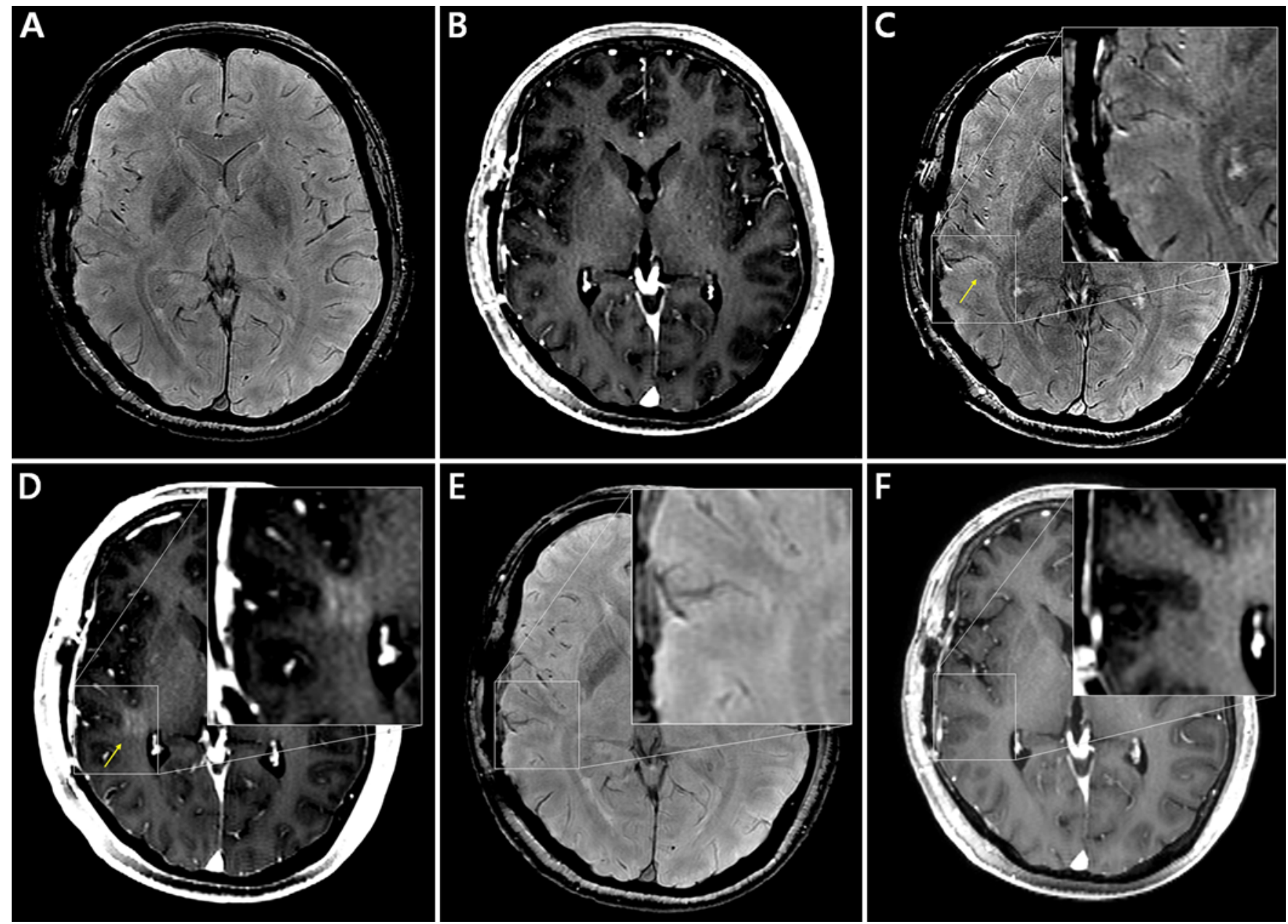

FIG. 3. BBBD on presonication and postsonication axial MRI in patient 6 during the third $(A-D)$ and fourth ( $E$ and $F)$ cycles. A-D: T1-weighted (A) and T2*-weighted (B) presonication images. T1-weighted (C) and T2*-weighted (D) postsonication images. Yellow arrows in panels $C$ and $D$ indicate the change in signal intensity with 9 visible spots along the sonication area of $1 \mathrm{~cm}^{3}$. $\mathrm{E}$ and F: T1-weighted $(\mathrm{E})$ and $\mathrm{T} 2^{*}$-weighted $(\mathrm{F})$ presonication images. One month after BBBD (i.e., 1 month after the third cycle), the visible spots disappeared. Figure is available in color online only.

cutoff value of the spectral dose associated with BBBD was 8.23 AU.

None of the 6 patients experienced any adverse events, such as intracerebral hemorrhage, edema, or newly enhancing lesions, at the sonicated areas during the 6 cycles of treatment; all had radiologically stable disease. One patient exhibited adverse events during the treatment, but these events were not directly related to BBBD. Patient 6 had a CTCAE (Common Terminology Criteria for Adverse Events) grade 2 hematological adverse event that was related to TMZ, but none of the other patients experienced any serious TMZ-related neurotoxicity or clinically adverse event.

\section{Discussion}

\section{Rationale of BBBD With MRgFUS for GBM}

To date, the BBB has been an obstacle in the treatment of intracranial tumors. As it imposes size and biochemical restrictions on the passage of molecules, lipid-soluble drugs smaller than $400 \mathrm{Da}$ can move across the BBB,
TABLE 3. The number of BBBD targets and BBBD confirmed on postsonication MRI

\begin{tabular}{lccccc}
\hline $\begin{array}{c}\text { Patient } \\
\text { No. }\end{array}$ & $\begin{array}{c}\text { Total No. } \\
\text { of Targets }\end{array}$ & $\begin{array}{c}\text { BBBD on } \\
\text { T1WI }\end{array}$ & $\begin{array}{c}\text { BBBD on } \\
\text { T2*WI }\end{array}$ & $\begin{array}{c}\text { BBBD on } \\
\text { T1WI \& T2*WI }\end{array}$ & $\begin{array}{c}\% \text { of } \\
\text { BBBD }\end{array}$ \\
\hline 1 & 22 & 19 & 16 & 21 & 95.5 \\
\hline 2 & 29 & 27 & 26 & 27 & 93.1 \\
\hline 3 & 24 & 17 & 5 & 17 & 70.8 \\
\hline $4 \dagger$ & 12 & 10 & 3 & 11 & 91.7 \\
\hline 5 & 29 & 29 & 21 & 29 & 100 \\
\hline 6 & 29 & 29 & 22 & 29 & 100 \\
\hline Total & 145 & 131 & 93 & 134 & 92.4 \\
\hline
\end{tabular}

$\mathrm{T} 2{ }^{*} \mathrm{WI}=\mathrm{T}^{*}$-weighted imaging.

† This patient had a GBM in the left parietal area and exhibited a minor personality issue before enrollment. The patient dropped out of the study prior to the fourth cycle of BBBD. 
TABLE 4. Sonication parameters

\begin{tabular}{|c|c|c|c|c|c|c|}
\hline Patient No. & Cycle 1 & Cycle 2 & Cycle 3 & Cycle 4 & Cycle 5 & Cycle 6 \\
\hline \multicolumn{7}{|l|}{1} \\
\hline Ramp sonication power, $\mathrm{W}$ & $13.0 \pm 4.24$ & $9.75 \pm 1.06$ & $5.5 \pm 0.57$ & $5.5 \pm 1.32$ & $8.0 \pm 2.31$ & $5.75 \pm 0.96$ \\
\hline 1st sonication power, W & $6.50 \pm 2.12$ & $4.5 \pm 1.32$ & $4.0 \pm 1.37$ & $3.17 \pm 0.76$ & $4.25 \pm 0.96$ & $2.63 \pm 0.85$ \\
\hline Spectral dose, AU & $1.03 \pm 0.53$ & $11.87 \pm 3.06$ & $13.87 \pm 3.24$ & $10.14 \pm 12.96$ & $7.39 \pm 1.86$ & $9.74 \pm 2.4$ \\
\hline \multicolumn{7}{|l|}{2} \\
\hline Ramp sonication power, $\mathrm{W}$ & $14.0 \pm 3.65$ & $11.60 \pm 4.39$ & $12.0 \pm 3.29$ & $11.9 \pm 1.8$ & $10.4 \pm 3.57$ & $10.2 \pm 3.49$ \\
\hline 1st sonication power, W & $6.63 \pm 1.60$ & $6.0 \pm 2.0$ & $6.5 \pm 1.29$ & $6.13 \pm 0.85$ & $5.5 \pm 1.5$ & $5.4 \pm 0.82$ \\
\hline Spectral dose, AU & $9.11 \pm 2.95$ & $8.75 \pm 1.56$ & $12.75 \pm 1.35$ & $14.48 \pm 5.56$ & $11.21 \pm 1.33$ & $11.16 \pm 2.98$ \\
\hline \multicolumn{7}{|l|}{3} \\
\hline Ramp sonication power, W & $20.67 \pm 3.06$ & $19.83 \pm 4.25$ & $19.0 \pm 2.0$ & $14.5 \pm 1.91$ & $15.83 \pm 1.93$ & $16.4 \pm 0.89$ \\
\hline 1st sonication power, W & $8.67 \pm 1.15$ & $10.33 \pm 2.52$ & $10.75 \pm 1.5$ & $8.0 \pm 1.41$ & $9.67 \pm 1.40$ & $9.0 \pm 0.71$ \\
\hline Spectral dose, AU & $5.21 \pm 2.0$ & $10.47 \pm 2.54$ & $10.51 \pm 5.04$ & $6.73 \pm 3.32$ & $7.59 \pm 3.41$ & $10.41 \pm 1.54$ \\
\hline \multicolumn{7}{|l|}{4} \\
\hline Ramp sonication power, W & $10.33 \pm 2.89$ & $10.50 \pm 4.65$ & $11.6 \pm 2.26$ & & & \\
\hline 1st sonication power, W & $4.67 \pm 1.26$ & $6.13 \pm 2.39$ & $6.0 \pm 1.0$ & & & \\
\hline Spectral dose, AU & $8.15 \pm 0.74$ & $10.89 \pm 2.2$ & $7.96 \pm 1.93$ & & & \\
\hline \multicolumn{7}{|l|}{5} \\
\hline Ramp sonication power, W & $11.25 \pm 2.22$ & $12.4 \pm 3.42$ & $10.9 \pm 2.70$ & $12.75 \pm 2.78$ & $11.83 \pm 2.81$ & $11.82 \pm 2.74$ \\
\hline 1st sonication power, W & $5.75 \pm 1.04$ & $6.0 \pm 2.04$ & $5.30 \pm 1.25$ & $6.5 \pm 0.94$ & $5.88 \pm 1.54$ & $5.9 \pm 1.1$ \\
\hline Spectral dose, AU & $10.03 \pm 2.44$ & $7.74 \pm 2.07$ & $6.85 \pm 2.33$ & $8.4 \pm 1.72$ & $8.88 \pm 2.53$ & $7.63 \pm 2.19$ \\
\hline \multicolumn{7}{|l|}{6} \\
\hline Ramp sonication power, W & $8.38 \pm 0.75$ & $10.48 \pm 1.26$ & $10.88 \pm 2.02$ & $10.35 \pm 0.75$ & $9.43 \pm 1.01$ & $10.62 \pm 1.39$ \\
\hline 1st sonication power, W & $4.88 \pm 0.75$ & $5.7 \pm 0.84$ & $6.50 \pm 1.66$ & $5.8 \pm 0.57$ & $5.29 \pm 0.8$ & $6.15 \pm 1.12$ \\
\hline Spectral dose, AU & $13.38 \pm 2.63$ & $8.36 \pm 2.55$ & $11.29 \pm 1.85$ & $10.52 \pm 1.19$ & $10.59 \pm 3.59$ & $10.9 \pm 1.6$ \\
\hline
\end{tabular}

but large and hydrophilic molecules cannot. ${ }^{16}$ Therefore, chemotherapeutic agents with relatively large molecular sizes cannot penetrate the BBB. Even when these agents penetrate the BBB, the P-glycoprotein 1 (P-gp), a common multidrug-resistant protein in the BBB that is abundant in cancerous tissue, is responsible for their efflux. ${ }^{1} \mathrm{P}-\mathrm{gp}$ does not allow the chemotherapeutic agent to reach proper concentrations in the brain, reducing its effectiveness. A high dose of a chemotherapeutic agent for appropriate concentration in the brain increases the risk of damage to peripheral organs, and spatially nonspecific methods, such as convection-enhanced delivery or wafers, have adverse effects on healthy brain tissue. For this reason, there is no method to efficiently and safely control GBM or other metastatic tumors with an infiltrating nature. ${ }^{19}$

Patrick et al. first described BBBD in the periphery of high-intensity FUS lesions. ${ }^{17}$ Many subsequent studies have shown that very low-intensity ultrasound is required for BBBD without causing damage to surrounding brain tissue and that using MBs requires lower ultrasound intensity for BBBD. ${ }^{14}$ Since then, many studies have been conducted using MRgFUS as a noninvasive means of temporarily disrupting the $\mathrm{BBB}$.

MRgFUS disrupts the BBB by 2 mechanisms: disruption of tight junctions and induced transcytosis. ${ }^{18}$ The shear stress produced by cavitation and the microflow produced by oscillation of the MBs when sonication starts causes local shock waves. These mechanisms affect the sensitive proteins in endothelial cells, transforming the extracellular mechanical signals into intracellular biochemical signals. ${ }^{23}$ This leads to a decrease in the integrity of tight junctions of the BBB, an increase in caveolins (integral membrane proteins involved in receptor-independent endocytosis), and a decrease in P-gp. ${ }^{4}$ When the BBB was disrupted, chemotherapeutic agents not detected in the brain tissue were found to be up to 5.8 times higher in the sonicated area. Moreover, in animal models of tumor, BBBD was found to increase the animals' survival rate. ${ }^{5,8,10}$ In addition, BBBD is temporary and is usually restored after 4 hours. ${ }^{18}$

However, until now, noninvasive MRgFUS with MBs has not been used for the treatment of patients with malignant brain tumors. FUS was used in 2 recent studies for the treatment of brain tumors in humans. The first study was a quantification of the penetration of TMZ via MRgFUS. ${ }^{12}$ It was the first study to evaluate BBBD in humans quantitatively, and a 7.7-fold difference was observed between the sonicated and unsonicated areas in 1 patient whose TMZ levels were measured. However, the aim of that study was not for actual treatment but to evaluate the efficacy of BBBD for the penetration of TMZ in brain tissue. Another BBBD study used an implanted FUS device for the treatment of recurrent GBM. In areas treated with low-intensity FUS, the tumor was well controlled, and, if BBBD was successful, the survival period was longer. However, implantation of a device requires surgery, and a target cannot 
be selected for an implanted FUS. ${ }^{9}$ Therefore, the present study was meaningful in that MRgFUS was repeatedly applied to the selected target in combination with a standard chemotherapy protocol.

\section{Safety and Feasibility of Multiple BBBD Trials in Patients With GBM}

After BBBD, a $3 \times 3$ grid of 9 spots was seen on T2*weighted/GRE or T1-weighted images; $90.3 \%$ of BBBD targets were seen as high signal spots on T1-weighted images, and $64.1 \%$ were seen as dark spots on T2*-weighted/ GRE images. An average of $62.1 \%$ of targets seen on T1weighted images were also seen on T2*-weighted/GRE images. The ratios of the targets at which BBBD was seen on T1-weighted images to that seen on T2*-weighted/GRE images were different among patients. The percentage of the spots on T2*-weighted/GRE images was lower than average in patients 3 and 4, with only $20.8 \%$ and $16.7 \%$, respectively, compared with $90 \%$ or more in other patients. Although the sensitivity of T1-weighted images to BBBD was higher than that of $\mathrm{T} 2 *$-weighted/GRE images and T2*-weighted/GRE images did not reflect BBBD well in some patients, there were some lesions that were only visible on T2*-weighted/GRE images and not on T1-weighted images. Therefore, both MRI sequences should be used in determining BBBD.

Usually, hypointensities on T2*-weighted/GRE images indicate microhemorrhages or calcifications. However, these spots were found to recover usually within 6-24 hours in other studies in which the same spots were seen at BBB disruption sites. ${ }^{13}$ In this study, as in previous studies, presonication MRI 2-3 days before the next procedure showed no evidence of hemorrhage in the BBBD site. In general, the signal of microhemorrhages on T2*-weighted/ GRE images became more intense over time and persisted for several weeks. ${ }^{2}$ Therefore, the dark signals of $\mathrm{T} 2{ }^{*}$ weighted/GRE images that disappeared over time in this study were thought to be dye leakage or venule dilatation.

Despite repeated BBBD, there were no adverse events such as hemorrhage, edema, or neurological deficits during the entire 6-cycle period for 5 patients (3-cycle period for the patient who was dropped from the study). BBBD adjacent to eloquent areas was performed in 5 patients in 28 trials. However, none of the patients showed cognition change, emotional change, or neurological deficit related to injury of these areas.

This study showed that BBBD can be achieved safely and repeatedly. To justify the benefit of additional BBBD with standard adjuvant chemotherapy for GBM, further study with a longer follow-up period is needed. This study was limited in that it was difficult to quantitate the increase in TMZ concentration due to BBBD. The purpose of this study was to confirm BBBD with gadolinium by comparing the 9 grid points on presonication and postsonication MRI by visual assessment. Another important goal was the safety and feasibility of multiple MRgFUS $\mathrm{BBB}$ disruptions for future application of this technique in practice. However, the imaging methods as used in this study were not sufficiently sensitive to quantify BBBD. Therefore, future investigation will be focused on which method is proper for quantification of BBBD: dynamic contrast-enhanced T1-weighted imaging, quantification of susceptibility mapping, T1 mapping, or T2* mapping.

\section{Technical Notes on BBBD}

In general, the mean half-life of Definity is 1.3 minutes, and it remains in the body for up to 10 minutes; thereafter, it is rarely found in the body. Therefore, it is better to perform sonication within 10 minutes after injection. Considering the average time from the start of MB injection to the start of sonication, between sonications, and the average number of sonications per target, 4 sessions of sonication in the target takes more than 10 minutes. Therefore, it is necessary to reach a sufficient spectral dose in 3 sessions of sonication. Otherwise, the number of targets would be reduced, as another injection of MBs should be used at the target. Of the sonications that were stopped earlier than 30 seconds, the majority were due to either a too-high or too-low sonication power. Therefore, to reach a sufficient spectral dose for BBBD within a limited time, it is important to start the sonication by finding an appropriate sonication power through ramp sonication.

For repeated BBBD, the results of the previous sonication session can be referred to in order to determine the proper sonication and ramp sonication power. Given that there were no significant differences in the ramp sonication power and start sonication power between cycles in the same patient, it is possible to predict how much spectral dose the sonication power will bring in the next session. However, there was a difference in sonication powers among patients. Even if sonication was performed using the same power, the bubble activity and spectral dose were different for each patient. For example, in patient 3, the sonication power was almost twice that in other patients, but the spectral dose was similar to or slightly lower than that of other patients. However, this patient's skull density, known as a factor affecting the ultrasound energy transfer through the skull, was similar to that in the other patients. ${ }^{3}$ Therefore, further studies on the factors affecting the relationship between sonication power and bubble activity are needed.

The determination of the first sonication power is assisted through ramp sonication, but there may be some difficulties in determining the appropriate sonication power. The amount of sonication power immediately before can be helpful. Sonication was repeated 10 times in succession with the same power. The mean time between sonications was $174.4 \pm 43.13$ seconds, and the spectral dose decreased to a mean of $44.5 \% \pm 27.6 \%$. In contrast, the number of cases in which the sonication power was increased by 1 in the following consecutive sonication was 63 . The mean time between sonications was $145.30 \pm 20.41$ seconds, and spectral dose was increased to $24.7 \% \pm 9.1 \%$. Although there were differences between patients, it might be helpful to determine the power by referring to the fact that there was about a $45 \%$ decrease or $25 \%$ increase at the same power or a power increase by 1 in the next sonication.

There were 8 cases of sonication with the same MB after target movement. The mean time spent on target movement was $225.75 \pm 56.32$ seconds, and the mean time between the start of sonication in the second target and $\mathrm{MB}$ injection was $384.13 \pm 74.24$ seconds. The spectral 
dose of sonication in the second target was $3.38 \pm 2.24$ AU. Sonication was performed once before target movement in 4 cases and twice in 4 cases. The spectral doses of sonication in the second target were $3.91 \pm 1.84$ and $2.85 \pm 2.74 \mathrm{AU}$, respectively. These figures were low for efficient BBBD. However, in 1 case, the spectral dose of sonication in the second target after 2 sonications and target movement was more than 6 . Therefore, if enough spectral dose is obtained from the first sonication on the first target, BBBD on the second target will be efficiently performed by moving quickly and giving enough power to the second target.

\section{Technical Issues of BBBD}

MRgFUS has many advantages. MRgFUS allowed us to select the target location and target size while adjusting the direction of ultrasound. In this study, we fixed the target size as $1 \mathrm{~cm}^{3}$ and set the target. Since we performed sonication along the white matter near the tumor margin where recurrence mainly occurs, BBBD was possible without significant restrictions. From experience, due to low frequency, it is possible to approach any target in the brain, unless they are very close to the skull, without much reflection at the soft tissue-skull boundary if the frame is fixed so that the target is close to the center of the frame.

Another type of device for FUS is an implantable one. Surgery is needed to implant the device, and the direction of sonication is fixed and limited. However, for MRgFUS, surgery is not required and setting the target at the desired location is possible. Using intraoperative MRI, the damage in surrounding tissues and BBBD can be identified. Moreover, through real-time acoustic feedback and power modulation, the opening power required to safely open the BBB can be controlled and adapted. MRgFUS has some limitations, including the need for preprocedural removal of hair and fixing the frame. Fixing the frame once a month in a similar position might be a great burden for the patient. Although submillimeter accuracy is required, fixing the frame could cause great emotional stress for the patient, so it would be necessary to consider other methods of accuracy.

\section{Future Perspectives of BBBD for Brain Tumors}

This study is meaningful in that it is the first study to perform repetitive MRgFUS at the same target with a standard chemotherapy protocol for malignant brain tumors. This study also showed that repeatedly performed temporary BBBD using MRgFUS is feasible and safe. It offers the potential for additional studies on chemotherapeutic agents, targeted therapy, and immunotherapy that could not be used due to the BBB.

\section{Conclusions}

BBBD with MRgFUS can be performed accurately and repeatedly without any adverse effects. To justify the benefits of BBBD with standard adjuvant chemotherapy for GBM, a longer follow-up period is required. This study would allow us to perform additional studies on other therapeutic agents that could not be used due to the BBB.

\section{Acknowledgments}

This study was supported by a grant from the Korea Health Technology R\&D Project through the Korea Health Industry Development Institute (KHIDI), funded by the Ministry of Health \& Welfare, Republic of Korea (grant no. HI19C0060). We would like to thank Jung Il Lee, MD (Samsung Medical Center), Jeong Hoon Kim, MD (Asan Medical Center), and Jong Hee Chang, MD (Severance Hospital), for their help in recruiting the patients. We would also like to thank Eun Jung Kweon, RN, for her great assistance in this study.

\section{References}

1. Adkins CE, Mittapalli RK, Manda VK, Nounou MI, Mohammad AS, Terrell TB, et al: P-glycoprotein mediated efflux limits substrate and drug uptake in a preclinical brain metastases of breast cancer model. Front Pharmacol 4:136, 2013

2. Ayaz M, Boikov AS, Haacke EM, Kido DK, Kirsch WM: Imaging cerebral microbleeds using susceptibility weighted imaging: one step toward detecting vascular dementia. J Magn Reson Imaging 31:142-148, 2010

3. Chang WS, Jung HH, Zadicario E, Rachmilevitch I, Tlusty T, Vitek S, et al: Factors associated with successful magnetic resonance-guided focused ultrasound treatment: efficiency of acoustic energy delivery through the skull. J Neurosurg 124:411-416, 2016

4. Deng J, Huang Q, Wang F, Liu Y, Wang Z, Wang Z, et al: The role of caveolin-1 in blood-brain barrier disruption induced by focused ultrasound combined with microbubbles. J Mol Neurosci 46:677-687, 2012

5. Fan CH, Ting CY, Chang YC, Wei KC, Liu HL, Yeh CK: Drug-loaded bubbles with matched focused ultrasound excitation for concurrent blood-brain barrier opening and braintumor drug delivery. Acta Biomater 15:89-101, 2015

6. Hochberg FH, Pruitt A: Assumptions in the radiotherapy of glioblastoma. Neurology 30:907-911, 1980

7. Hynynen K, Jolesz FA: Demonstration of potential noninvasive ultrasound brain therapy through an intact skull. Ultrasound Med Biol 24:275-283, 1998

8. Hynynen K, McDannold N, Vykhodtseva N, Jolesz FA: Noninvasive MR imaging-guided focal opening of the bloodbrain barrier in rabbits. Radiology 220:640-646, 2001

9. Idbaih A, Canney M, Belin L, Desseaux C, Vignot A, Bouchoux G, et al: Safety and feasibility of repeated and transient blood-brain barrier disruption by pulsed ultrasound in patients with recurrent glioblastoma. Clin Cancer Res 25:3793-3801, 2019

10. Kinoshita M, McDannold N, Jolesz FA, Hynynen K: Noninvasive localized delivery of Herceptin to the mouse brain by MRI-guided focused ultrasound-induced blood-brain barrier disruption. Proc Natl Acad Sci U S A 103:11719-11723, 2006

11. Lipsman N, Meng Y, Bethune AJ, Huang Y, Lam B, Masellis M, et al: Blood-brain barrier opening in Alzheimer's disease using MR-guided focused ultrasound. Nat Commun 9:2336, 2018

12. Mainprize T, Lipsman N, Huang Y, Meng Y, Bethune A, Ironside $\mathrm{S}$, et al: Blood-brain barrier opening in primary brain tumors with non-invasive MR-guided focused ultrasound: a clinical safety and feasibility study. Sci Rep 9:321, 2019

13. O'Reilly MA, Hough O, Hynynen K: Blood-brain barrier closure time after controlled ultrasound-induced opening is independent of opening volume. J Ultrasound Med 36:475483, 2017

14. O'Reilly MA, Hynynen K: Blood-brain barrier: real-time feedback-controlled focused ultrasound disruption by using an acoustic emissions-based controller. Radiology 263:96106,2012 
15. Ostrom QT, Gittleman H, Fulop J, Liu M, Blanda R, Kromer $\mathrm{C}$, et al: CBTRUS statistical report: Primary brain and central nervous system tumors diagnosed in the United States in 2008-2012. Neuro Oncol 17 (Suppl 4):iv1-iv62, 2015

16. Pandey PK, Sharma AK, Gupta U: Blood brain barrier: an overview on strategies in drug delivery, realistic in vitro modeling and in vivo live tracking. Tissue Barriers 4:e1129476, 2015

17. Patrick JT, Nolting MN, Goss SA, Dines KA, Clendenon JL, Rea MA, et al: Ultrasound and the blood-brain barrier. Adv Exp Med Biol 267:369-381, 1990

18. Sheikov N, McDannold N, Sharma S, Hynynen K: Effect of focused ultrasound applied with an ultrasound contrast agent on the tight junctional integrity of the brain microvascular endothelium. Ultrasound Med Biol 34:1093-1104, 2008

19. Song KH, Harvey BK, Borden MA: State-of-the-art of microbubble-assisted blood-brain barrier disruption. Theranostics 8:4393-4408, 2018

20. Stepanenko AA, Chekhonin VP: Recent advances in oncolytic virotherapy and immunotherapy for glioblastoma: a glimmer of hope in the search for an effective therapy? Cancers (Basel) 10:10, 2018

21. Stupp R, Hegi ME, Mason WP, van den Bent MJ, Taphoorn MJ, Janzer RC, et al: Effects of radiotherapy with concomitant and adjuvant temozolomide versus radiotherapy alone on survival in glioblastoma in a randomised phase III study: 5-year analysis of the EORTC-NCIC trial. Lancet Oncol 10:459-466, 2009

22. Stupp R, Mason WP, van den Bent MJ, Weller M, Fisher B, Taphoorn MJ, et al: Radiotherapy plus concomitant and adjuvant temozolomide for glioblastoma. N Engl J Med 352:987-996, 2005
23. Yang FY, Fu WM, Chen WS, Yeh WL, Lin WL: Quantitative evaluation of the use of microbubbles with transcranial focused ultrasound on blood-brain-barrier disruption. Ultrason Sonochem 15:636-643, 2008

\section{Disclosures}

Dr. J. W. Chang received funding from InSightec for this clinical trial. Mr. Rachmilevitch and Dr. Zadicario are employees of InSightec.

\section{Author Contributions}

Conception and design: JW Chang, Choi, Zadicario. Acquisition of data: JW Chang, Park, Kim. Analysis and interpretation of data: Park, Rachmilevitch. Drafting the article: Park, Rachmilevitch. Critically revising the article: JW Chang, Park. Reviewed submitted version of manuscript: JW Chang, Jung, WS Chang, Choi, Zadicario. Approved the final version of the manuscript on behalf of all authors: JW Chang. Statistical analysis: Park. Administrative/technical/material support: Kim, Jung, WS Chang, Rachmilevitch, Zadicario. Study supervision: JW Chang.

\section{Correspondence}

Jin Woo Chang: Yonsei University College of Medicine, Seoul, Republic of Korea. jchang@yuhs.ac. 